\title{
Studies on Maturity Indicators of Leucaena leucocephala in Northern India
}

\author{
Pankaj Lavania ${ }^{1 *}$, Ashish Tiwari ${ }^{2}$ and Naresh Kumar ${ }^{3}$ \\ ${ }^{1}$ Rani Lakshmi Bai Central Agricultural University Jhansi-Uttar Pradesh -284003, India \\ ${ }^{2}$ Department of forestry, D.S.B. Campus, Kumaun University, Nanital, \\ Uttarakhand-263001, India \\ ${ }^{3}$ Central Agroforestry Research Institute, Jhansi-Uttar Pradesh-284003, India \\ *Corresponding author
}

\section{A B S T R A C T}

\begin{tabular}{|l|}
\hline Ke y w or d s \\
Moisture content, \\
Pod colour, Seed \\
colour, Seed \\
germination, Seed \\
maturity
\end{tabular}

\section{Introduction}

Leucaena leucocephala (Mimosoideae), commonly known as subabool is a mediumsized, multipurpose, deciduous tree species. It is a valuable source of pulpwood, small timber, fuel wood, fodder and green manure. It is a tree of the tropics and subtropics and within this region grows up to an elevation of about $500 \mathrm{~m}$. Genus Leucaena consists of 22 identified species (Brewbaker, 2016). Botanical literature claims a sum of 55 species. The only valid ones appear to be $L$. collinsii, $L$. diversifolia, $L$. esculenta, $L$.
Leucaena leucocephala is a medium-sized, multipurpose tree which is a valuable source of pulpwood, small timber, fuel wood, fodder and green manure. Poor and delayed seed germination is reported due to seed collection at improper maturity stage coupled with physical dormancy. Hence, the present study was carried out to determine the exact maturity stage for seed collection at three locations of northern India i.e. Agra $\left(S_{1}\right)$, Mathura $\left(S_{2}\right)$ and Hathrus $\left(S_{3}\right)$. Among the different pod/seed collection periods, $1^{\text {st }}$ week of June $\left(8^{\text {th }}\right.$ collection)was found best across all the sites as maximum germination $58.7 \pm 0.25 \%$ at $\mathrm{S}_{1}, 55.1 \pm 0.43 \%$ at $\mathrm{S}_{2}, 54.2 \pm 0.28 \%$ at $\mathrm{S}_{3}$ was recorded during this stage. The results revealed that moisture content decreased with advancement of maturity. The brown colour of pod and dark brown colour of seed were identified as seed maturity indicators. 
collection of seeds at proper maturity stage and pre-treatments can give good germination, and more vigorous seedlings of Leucaena leucocephala can be obtained by inoculating with $R$. irregularis (Kumar, 2018). Thus, the knowledge of proper stage and time of maturity of seeds is essential for collection of abundant quantity of healthy and vigorous seeds. Keeping in view the importance of species and the lack of information on maturity indicators of this species in semi-arid conditions of northern India, the present study was carried out.

\section{Materials and Methods}

The present study was conducted in Agra $\left(\mathrm{S}_{1}\right)$, Mathura $\left(\mathrm{S}_{2}\right)$ and Hathrus $\left(\mathrm{S}_{3}\right)$ sites of northern India which lies between $27^{\circ} 10^{\prime} \mathrm{N}$ to $26^{\circ}$ ' $4 \mathrm{~N}$ ' latitude and $78^{\circ} 02^{\prime} \mathrm{E}$ to $79^{\circ} 7^{\prime} \mathrm{E}$ longitude between elevation 165 and $179.8 \mathrm{~m}$ amsl. Five trees with clear bole, compact crown, having fair number of pods were selected for the study at each site. The pods of Leucaena leucocephala were collected from the all three sites at different dates. First pods collection was made on $2^{\text {rd }}$ week of February, 2004 and subsequent collections were made at bi-weekly intervals, until the completion of natural seed fall. Pods from different tree species were separately sealed in plastic bags and brought to the laboratory.

Before the seed extraction and cleaning, pod parameters like length, width, moisture content (\%), weight of 100 pods, number of pod per $100 \mathrm{~g}$ were measure for each collection dates. The size dimensions were recorded with manual Vernier's caliper and digital electronic balance was used for weighing of pod weight. Moisture content (\%) was determined on fresh weight basis by drying (over drying) the material at $103 \pm 2{ }^{\circ} \mathrm{C}$ for $16 \pm 1 \mathrm{hrs}$ (ISTA, 1981). The pods were dried for 4 to 5 days and thereafter beaten with a stick and cleaned by winnowing to release seeds. After the process of seed extraction and cleaning, 3 replicates of 10 seeds were measured for seed length, width and size using Varnier's calliper and weight parameters like number of seed/g, weight of 100 seeds. Moisture content was recorded on fresh weight basis by drying seeds at $103 \pm 1^{\circ} \mathrm{C}$ for $16 \pm 1 \mathrm{hrs}$ (ISTA, 1981) and moisture content was calculated as:

\section{Moisturecontent \%=}

$\frac{\text { Fresh weight }- \text { Dry weight }}{\text { Fresh weight }} \times 100$

Fresh weight

The seeds were surface sterilized with 0.1 $\mathrm{HgCl}_{2}$. Seeds were rinsed thoroughly to remove traces of mercuric chloride before placing for germination. For germination, 3 replicates of 100 seeds each were used. The germination was carried out in Petri dish at laboratory (room temperature) for each collection date. Germination was counted when visible radicle develops. Germination percent was calculated as follow:

$$
\text { Germination }(\%)=\frac{\text { Number of germinated seed }(\text { vissible redicle of seeds })}{\text { Total number of seeds put in petridish }} \times 100
$$

Data recorded for different characters on different sites have been tested for their significance by using statistical technique of analysis of variance with Randomized block design suggested by Panse and Sukhatame (1961).

\section{Results and Discussion}

The results presented in Table 1 revealed that the pod colour of L. leucocephala changed from the green colour ( $2^{\text {nd }}$ week of February) to brown ( $1^{\text {st }}$ week of June) across all the collection sites. 
Table.1 Physical parameters of pods of Leucaena leucecephala over the collection period from February to Starting June in different sites

\begin{tabular}{|c|c|c|c|c|c|c|c|c|}
\hline Site & Date & $\begin{array}{l}\text { Day of } \\
\text { collection }\end{array}$ & Pod colours & $\begin{array}{l}\text { Pod length } \\
\text { (cm) }\end{array}$ & Pod width (cm) & Wt. of 100 pod (gm) & No. of pod/100 gm & $\begin{array}{l}\text { Pod moisture } \\
\text { content }(\%)\end{array}$ \\
\hline \multirow[t]{9}{*}{$\mathbf{S}_{1}$} & $20 / 2$ & $\mathrm{D}_{1}$ & Green & $18.7 \pm 0.24$ & $1.4 \pm 0.03$ & $258.1 \pm 1.15$ & $39.0 \pm 0.63$ & $52.9 \pm 0.44$ \\
\hline & $7 / 3$ & $\mathrm{D}_{2}$ & Green & $19.2 \pm 0.20$ & $1.8 \pm 0.05$ & $350.7 \pm 1.29$ & $30.1 \pm 0.31$ & $55.2 \pm 0.75$ \\
\hline & $22 / 3$ & $\mathrm{D}_{3}$ & Light Green & $21.3 \pm 0.23$ & $1.8 \pm 0.06$ & $501.5 \pm 1.16$ & $19.4 \pm 0.37$ & $60.6 \pm 0.68$ \\
\hline & $6 / 4$ & $\mathrm{D}_{4}$ & $\begin{array}{l}\text { Light Green } \\
\text { Golden }\end{array}$ & $20.9 \pm 0.22$ & $1.8 \pm 0.06$ & $504.1 \pm 3.34$ & $25.4 \pm 0.37$ & $59.1 \pm 0.31$ \\
\hline & $21 / 4$ & $\mathrm{D}_{5}$ & $\begin{array}{c}\text { Light Yellow } \\
\text { Green }\end{array}$ & $20.4 \pm 0.57$ & $1.9 \pm 0.02$ & $511.1 \pm 2.30$ & $19.8 \pm 0.63$ & $58.0 \pm 0.52$ \\
\hline & $6 / 5$ & $\mathrm{D}_{6}$ & $\begin{array}{l}\text { Yellowish } \\
\text { Green with } \\
\text { Brown spot }\end{array}$ & $20.5 \pm 0.52$ & $1.98 \pm 0.02$ & $529.6 \pm 2.15$ & $24.1 \pm 0.48$ & $56.8 \pm 0.25$ \\
\hline & $21 / 5$ & $\mathrm{D}_{7}$ & Light Brown & $20.5 \pm 0.54$ & $2.0 \pm 0.04$ & $526.0 \pm 7.72$ & $26.3 \pm 0.47$ & $50.2 \pm 0.43$ \\
\hline & $5 / 6$ & $\mathrm{D}_{8}$ & Brown & $20.3 \pm 0.37$ & $2.0 \pm 0.07$ & $303.0 \pm 1.57$ & $33.2 \pm 0.99$ & $40.6 \pm 0.25$ \\
\hline & & Mean & & 20.26 & 1.85 & 435.51 & 27.16 & 54.2 \\
\hline \multirow[t]{9}{*}{$\mathbf{S}_{\mathbf{2}}$} & $21 / 2$ & $\mathrm{D}_{1}$ & Green & $18.5 \pm 0.24$ & $1.3 \pm 0.03$ & $250.6 \pm 2.29$ & $42.9 \pm 1.13$ & $54.5 \pm 0.32$ \\
\hline & $8 / 3$ & $\mathrm{D}_{2}$ & Green & $19.2 \pm 0.16$ & $1.7 \pm 0.06$ & $352.2 \pm 1.78$ & $30.7 \pm 0.42$ & $57.0 \pm 0.36$ \\
\hline & $23 / 3$ & $\mathrm{D}_{3}$ & Light Green & $20.9 \pm 0.24$ & $1.8 \pm 0.03$ & $497.0 \pm 0.79$ & $21.7 \pm 0.41$ & $63.4 \pm 0.31$ \\
\hline & $7 / 4$ & $\mathrm{D}_{4}$ & $\begin{array}{l}\text { Light Green } \\
\text { Golden }\end{array}$ & $20.9 \pm 0.22$ & $1.8 \pm 0.03$ & $500.8 \pm 2.87$ & $24.8 \pm 0.25$ & $61.5 \pm 0.35$ \\
\hline & $22 / 4$ & $\mathrm{D}_{5}$ & $\begin{array}{l}\text { Light Yellow } \\
\text { Green }\end{array}$ & $20.6 \pm 0.41$ & $1.9 \pm 0.03$ & $498.8 \pm 2.47$ & $21.6 \pm 0.52$ & $59.9 \pm 0.47$ \\
\hline & $7 / 5$ & $\mathrm{D}_{6}$ & $\begin{array}{l}\text { Yellowish } \\
\text { Green with } \\
\text { Brown spot }\end{array}$ & $20.5 \pm 0.41$ & $1.8 \pm 0.03$ & $457.4 \pm 5.88$ & $25.4 \pm 0.52$ & $59.0 \pm 0.37$ \\
\hline & $22 / 5$ & $\mathrm{D}_{7}$ & Light Brown & $20.4 \pm 0.39$ & $1.8 \pm 0.04$ & $395.1 \pm 0.70$ & $29.2 \pm 0.49$ & $53.0 \pm 0.83$ \\
\hline & $6 / 6$ & $\mathrm{D}_{8}$ & Brown & $20.3 \pm 0.35$ & $1.9 \pm 0.04$ & $295.7 \pm 0.66$ & $36.7 \pm 0.41$ & $39.2 \pm 0.41$ \\
\hline & & Mean & & 20.18 & 2.0 & 405.95 & 29.08 & 56.1 \\
\hline
\end{tabular}


Int.J.Curr.Microbiol.App.Sci (2018) 7(12): 3636-3643

\begin{tabular}{|c|c|c|c|c|c|c|c|c|}
\hline \multirow[t]{4}{*}{$\mathbf{S}_{3}$} & $22 / 2$ & $\mathrm{D}_{1}$ & Green & $18.1 \pm 0.25$ & $1.3 \pm 0.04$ & $253.3 \pm 4.67$ & $44.8 \pm 1.16$ & $56.2 \pm 0.41$ \\
\hline & $9 / 3$ & $\mathrm{D}_{2}$ & Green & $19.5 \pm 0.16$ & $1.6 \pm 1.03$ & $358.7 \pm 1.96$ & $33.5 \pm 0.45$ & $59.0 \pm 0.27$ \\
\hline & $24 / 3$ & $\mathrm{D}_{3}$ & Light Green & $20.1 \pm 0.57$ & $1.7 \pm 0.03$ & $485.6 \pm 1.03$ & $27.0 \pm 0.30$ & $65.9 \pm 0.40$ \\
\hline & $8 / 4$ & $\mathrm{D}_{4}$ & $\begin{array}{l}\text { Light Green } \\
\text { Golden }\end{array}$ & $20.8 \pm 0.41$ & $1.8 \pm 0.03$ & $486.9 \pm 0.76$ & $28.9 \pm 0.31$ & $62.2 \pm 0.47$ \\
\hline & $23 / 4$ & $\mathrm{D}_{5}$ & $\begin{array}{l}\text { Light Yellow } \\
\text { Green }\end{array}$ & $19.7 \pm 0.62$ & $1.9 \pm 0.03$ & $490.0 \pm 1.18$ & $24.9 \pm 0.31$ & $61.1 \pm 0.47$ \\
\hline & $8 / 5$ & $\mathrm{D}_{6}$ & $\begin{array}{l}\text { Yellowish } \\
\text { Green with } \\
\text { Brown spot }\end{array}$ & $20.6 \pm 0.51$ & $1.8 \pm 0.02$ & $527.1 \pm 2.68$ & $28.2 \pm 0.39$ & $60.2 \pm 0.28$ \\
\hline & $23 / 5$ & $\mathrm{D}_{7}$ & Light Brown & $20.4 \pm 0.42$ & $1.9 \pm 0.03$ & $537.9 \pm 1.60$ & $29.2 \pm 0.39$ & $54.5 \pm 0.28$ \\
\hline & $7 / 6$ & $\mathrm{D}_{8}$ & Brown & $20.4 \pm 0.33$ & $1.8 \pm 0.05$ & $286.0 \pm 4.01$ & $37.6 \pm 0.34$ & $42.7 \pm 0.35$ \\
\hline & & Mean & & 19.98 & 1.7 & 428.19 & 31.76 & 57.73 \\
\hline \multicolumn{3}{|c|}{$\begin{array}{l}\text { Average of Across all } \\
\text { site }\end{array}$} & & 20.14 & 1.85 & 423.22 & 29.33 & 56.01 \\
\hline \multicolumn{3}{|c|}{ SEm \pm for site } & & 0.19 & 0.21 & 4.66 & 0.28 & 0.22 \\
\hline \multicolumn{3}{|c|}{ F-test } & & NS & NS & $*$ & $*$ & $*$ \\
\hline \multicolumn{4}{|c|}{ CD } & - & - & 9.22 & 0.55 & 0.44 \\
\hline \multicolumn{4}{|c|}{ SEm+ for Date/day } & 0.31 & 0.34 & 7.60 & 0.45 & 0.36 \\
\hline \multicolumn{4}{|c|}{ F-test } & $*$ & $*$ & $*$ & $*$ & $*$ \\
\hline \multicolumn{4}{|c|}{ CD for day } & 0.61 & 0.67 & 15.06 & 0.895 & 0.71 \\
\hline \multicolumn{4}{|c|}{ SEm+ for SxD } & 0.54 & 0.59 & 13.17 & 0.78 & 0.623 \\
\hline \multicolumn{4}{|c|}{ F-test } & NS & NS & $*$ & $*$ & $*$ \\
\hline \multicolumn{4}{|c|}{ CD for SxD } & - & - & 26.08 & 1.55 & 1.23 \\
\hline
\end{tabular}

NS- Non Significant, *- Significance at 5\% $(\mathrm{P}<0.05)$ 
Table.2 Physical parameters of seeds sites of Leucaena leucocephala over the collection period from end of February to Starting June in different sites

\begin{tabular}{|c|c|c|c|c|c|c|c|c|c|c|c|c|}
\hline$\stackrel{\infty}{\frac{\pi}{n}}$ & อัँ & 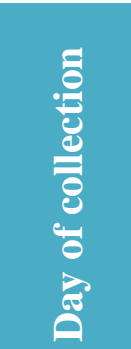 & 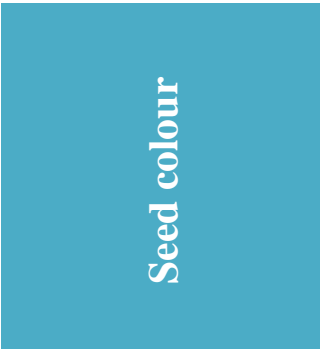 & 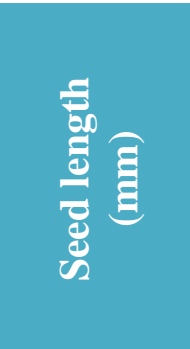 & 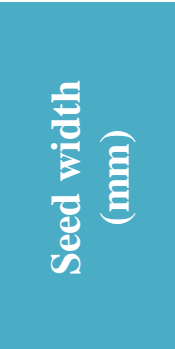 & 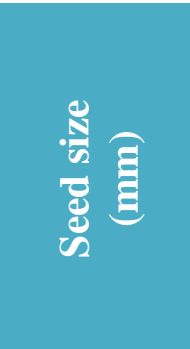 & 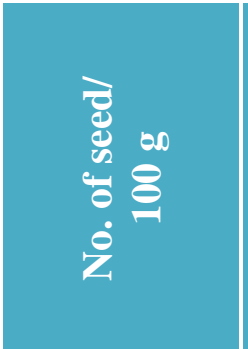 & 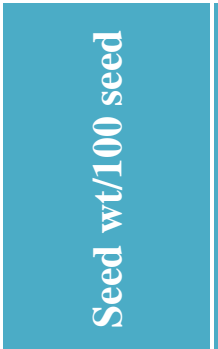 & 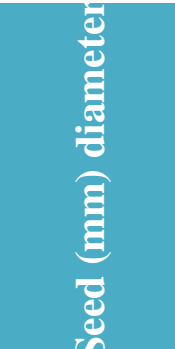 & 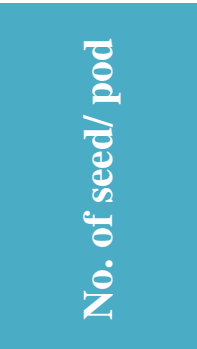 & 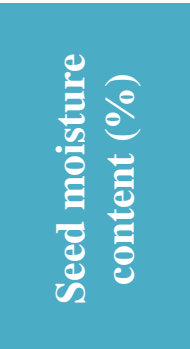 & 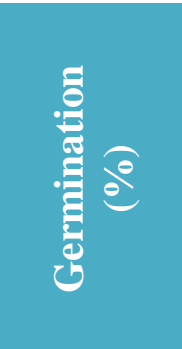 \\
\hline \multirow[t]{8}{*}{$\mathbf{S}_{1}$} & $20 / 2$ & $\mathrm{D}_{1}$ & Dark Green & $6.4 \pm 0.22$ & $3.7 \pm 0.15$ & $41.4 \pm 2.74$ & $1030.3 \pm 0.45$ & $88.7 \pm 0.22$ & $1.0 \pm 0.10$ & $22.6 \pm 0.54$ & $53.1 \pm 0.39$ & $0.0 \pm 0.0$ \\
\hline & $7 / 3$ & $\mathrm{D}_{2}$ & Light Green & $6.7 \pm 0.15$ & $4.2 \pm 0.13$ & $45.1 \pm 1.99$ & $1091.4 \pm 1.42$ & $113.8 \pm 0.21$ & $1.1 \pm 0.10$ & $23.6 \pm 0.34$ & $53.0 \pm 0.96$ & $0.0 \pm 0.0$ \\
\hline & $22 / 3$ & $\mathrm{D}_{3}$ & Bright Green & $7.0 \pm 0.28$ & $4.8 \pm 0.20$ & $49.6 \pm 3.62$ & $1124.2 \pm 8.84$ & $178.1 \pm 0.07$ & $1.6 \pm 0.16$ & $25.7 \pm 0.45$ & $50.9 \pm 0.52$ & $6.5 \pm 0.21$ \\
\hline & $6 / 4$ & $\mathrm{D}_{4}$ & Bright Green & $7.2 \pm 0.13$ & $4.8 \pm 0.20$ & $52.6 \pm 2.00$ & $813.1 \pm 6.62$ & $96.1 \pm 0.10$ & $1.5 \pm 0.16$ & $24.8 \pm 0.42$ & $48.3 \pm 0.35$ & $35.5 \pm 0.31$ \\
\hline & $21 / 4$ & $\mathrm{D}_{5}$ & $\begin{array}{c}\text { Yellowish Bright } \\
\text { Green }\end{array}$ & $7.6 \pm 0.16$ & $4.9 \pm 0.23$ & $58.0 \pm 2.45$ & $551.3 \pm 3.49$ & $180.7 \pm 0.12$ & $1.4 \pm 0.16$ & $23.5 \pm 1.16$ & $45.4 \pm 0.27$ & $49.1 \pm 0.42$ \\
\hline & $6 / 5$ & $\mathrm{D}_{6}$ & $\begin{array}{c}\text { Yellow Brownish } \\
\text { Green }\end{array}$ & $7.5 \pm 0.16$ & $4.8 \pm 0.20$ & $56.5 \pm 2.50$ & $1012.2 \pm 3.45$ & $150.3 \pm 0.13$ & $1.4 \pm 0.16$ & $22.8 \pm 1.02$ & $44.5 \pm 0.35$ & $52.0 \pm 0.52$ \\
\hline & $21 / 5$ & $\mathrm{D}_{7}$ & $\begin{array}{l}\text { Bright Light } \\
\text { Brown }\end{array}$ & $7.2 \pm 0.13$ & $4.7 \pm 0.15$ & $52.0 \pm 2.00$ & $1413.3 \pm 3.55$ & $126.3 \pm 0.14$ & $1.4 \pm 0.16$ & $22.1 \pm 0.92$ & $43.5 \pm 0.38$ & $56.3 \pm 0.22$ \\
\hline & & Mean & & 7.05 & 4.5 & 50.15 & 1121.4 & 12.34 & 1.38 & 23.3 & 47.6 & 32.27 \\
\hline \multirow[t]{7}{*}{$\mathbf{S}_{2}$} & $21 / 2$ & $\mathrm{D}_{1}$ & Dark Green & $6.1 \pm 0.23$ & $3.5 \pm 0.17$ & $37.7 \pm 2.83$ & $1033.2 \pm 1.56$ & $83.0 \pm 0.30$ & $1.0 \pm 0.10$ & $24.2 \pm 0.89$ & $53.1 \pm 0.32$ & $0.0 \pm 0.0$ \\
\hline & $8 / 3$ & $\mathrm{D}_{2}$ & Light Green & $6.2 \pm 0.20$ & $3.9 \pm 0.18$ & $38.8 \pm 2.47$ & $1097.7 \pm 1.58$ & $86.2 \pm 0.29$ & $1.1 \pm 0.10$ & $24.3 \pm 0.49$ & $54.0 \pm 0.23$ & $0.0 \pm 0.0$ \\
\hline & $23 / 3$ & $\mathrm{D}_{3}$ & Bright Green & $6.8 \pm 0.25$ & $4.5 \pm 0.17$ & $46.8 \pm 3.46$ & $1166.8 \pm 12.61$ & $92.0 \pm 0.11$ & $1.3 \pm 0.15$ & $25.6 \pm 0.50$ & $52.3 \pm 0.24$ & $5.0 \pm 0.44$ \\
\hline & $7 / 4$ & $\mathrm{D}_{4}$ & Bright Green & $7.1 \pm 0.23$ & $4.5 \pm 0.17$ & $50.9 \pm 3.16$ & $894.0 \pm 12.81$ & $138.0 \pm 0.11$ & $1.3 \pm 0.15$ & $24.5 \pm 0.43$ & $49.0 \pm 0.31$ & $30.8 \pm 0.49$ \\
\hline & $22 / 4$ & $\mathrm{D}_{5}$ & $\begin{array}{c}\text { Yellowish Bright } \\
\text { Green }\end{array}$ & $7.4 \pm 0.16$ & $4.5 \pm 0.22$ & $56.5 \pm 2.50$ & $556.8 \pm 2.35$ & $174.5 \pm 0.12$ & $1.2 \pm 0.13$ & $23.3 \pm 0.73$ & $47.1 \pm 0.41$ & $45.1 \pm 0.28$ \\
\hline & $7 / 5$ & $\mathrm{D}_{6}$ & $\begin{array}{c}\text { Yellow Brownish } \\
\text { Green }\end{array}$ & $7.2 \pm 0.25$ & $4.4 \pm 0.16$ & $52.6 \pm 2.00$ & $1034.9 \pm 2.30$ & $159.5 \pm 0.12$ & $1.4 \pm 0.16$ & $23.1 \pm 0.48$ & $46.3 \pm 0.53$ & $51.9 \pm 0.48$ \\
\hline & $22 / 5$ & $\mathrm{D}_{7}$ & Bright Light & $7.0 \pm 0.101$ & $4.4 \pm 0.16$ & $49.0 \pm 0.16$ & $1513.9 \pm 2.21$ & $137.5 \pm 0.30$ & $1.5 \pm 0.16$ & $22.2 \pm 0.47$ & $45.9 \pm 0.33$ & $52.1 \pm 0.38$ \\
\hline
\end{tabular}




\begin{tabular}{|c|c|c|c|c|c|c|c|c|c|c|c|c|}
\hline \multicolumn{13}{|c|}{ Brown } \\
\hline & $6 / 6$ & $\mathrm{D}_{8}$ & Dark Brown & $6.6 \pm 0.22$ & $4.4 \pm 0.16$ & $44.0 \pm 3.02$ & $1992.0 \pm 5.31$ & $51.9 \pm 0.10$ & $1.5 \pm 0.16$ & $20.7 \pm 0.34$ & $44.0 \pm 0.38$ & $55.1 \pm 0.43$ \\
\hline & & Mean & & 6.8 & 4.2 & 46.9 & 1161.2 & 11.53 & 1.28 & 23.48 & 48.96 & 30.01 \\
\hline $\mathbf{S}_{\mathbf{3}}$ & $22 / 2$ & $\mathrm{D}_{1}$ & Dark Green & $5.9 \pm 0.23$ & $3.4 \pm 0.16$ & $35.3 \pm 2.78$ & $1040.4 \pm 2.18$ & $83.0 \pm 0.31$ & $1.0 \pm 0.10$ & $24.7 \pm 0.56$ & $57.2 \pm 0.27$ & $0.0 \pm 0.0$ \\
\hline & $24 / 3$ & $\mathrm{D}_{3}$ & Bright Green & $6.5 \pm 0.17$ & $4.3 \pm 0.15$ & $42.5 \pm 2.17$ & $1117.1 \pm 9.93$ & $87.3 \pm 0.06$ & $1.3 \pm .15$ & $26.2 \pm 0.36$ & $56.9 \pm 0.39$ & $5.4 \pm 0.77$ \\
\hline & $8 / 4$ & $\mathrm{D}_{4}$ & Bright Green & $6.7 \pm 0.15$ & $4.4 \pm 0.16$ & $45.1 \pm 1.81$ & $942.1 \pm 2.60$ & $138.5 \pm 0.07$ & $1.3 \pm 0.15$ & $25.7 \pm 0.15$ & $49.8 \pm 0.44$ & $32.0 \pm 0.64$ \\
\hline & $23 / 4$ & $\mathrm{D}_{5}$ & $\begin{array}{c}\text { Yellowish Bright } \\
\text { Green }\end{array}$ & $7.2 \pm 0.25$ & $4.5 \pm 0.17$ & $52.4 \pm 3.53$ & $561.4 \pm 1.79$ & $170.5 \pm 0.12$ & $1.2 \pm 0.13$ & $23.5 \pm 0.69$ & $47.9 \pm 0.32$ & $46.2 \pm 0.55$ \\
\hline & $8 / 5$ & $\mathrm{D}_{6}$ & $\begin{array}{c}\text { Yellow Brownish } \\
\text { Green }\end{array}$ & $7.0 \pm 0.21$ & $4.4 \pm 0.16$ & $49.4 \pm 2.96$ & $1070.2 \pm 1.91$ & $150.5 \pm 0.12$ & $1.3 \pm 0.15$ & $23.4 \pm 0.70$ & $46.9 \pm 0.21$ & $52.2 \pm 0.73$ \\
\hline & $23 / 5$ & $\mathrm{D}_{7}$ & $\begin{array}{l}\text { Bright Light } \\
\text { Brown }\end{array}$ & $6.6 \pm 0.16$ & $4.3 \pm 0.15$ & $43.8 \pm 2.12$ & $1619.5 \pm 1.94$ & $119.5 \pm 0.13$ & $1.4 \pm 0.16$ & $22.0 \pm 0.58$ & $46.5 \pm 0.33$ & $54.0 \pm 0.57$ \\
\hline & $7 / 6$ & $\mathrm{D}_{8}$ & Dark Brown & $6.4 \pm 0.16$ & $4.3 \pm 0.15$ & $41.2 \pm 2.12$ & $1997.2 \pm 5.38$ & $50.9 \pm 0.11$ & $1.3 \pm 0.15$ & $20.6 \pm 0.37$ & $45.9 \pm 0.38$ & $54.2 \pm 0.28$ \\
\hline & & Mean & & 6.55 & 4.180 & 43.2 & 1168.6 & 11.06 & 1.24 & 23.83 & 50.8 & 30.5 \\
\hline \multicolumn{3}{|c|}{$\begin{array}{c}\text { Average of Across } \\
\text { all site } \\
\end{array}$} & & 6.8 & 4.29 & 46.75 & 1150.4 & 11.64 & 1.3 & 23.54 & 49.12 & \\
\hline \multicolumn{3}{|c|}{ F-test } & & $*$ & $*$ & $*$ & $*$ & NS & $*$ & $*$ & $*$ & $*$ \\
\hline \multicolumn{4}{|c|}{ CD } & 0.191 & 0.171 & 2.52 & 36.13 & - & 0.14 & NS & 0.40 & 0.38 \\
\hline \multicolumn{4}{|c|}{ SEm+ for Date/day } & 0.157 & 0.14 & 2.08 & 18.25 & 1.44 & 0.113 & 0.57 & 0.327 & 0.31 \\
\hline \multicolumn{4}{|c|}{ F-test } & $*$ & $*$ & $*$ & $*$ & $*$ & $*$ & * & $*$ & $*$ \\
\hline \multicolumn{4}{|c|}{ CD for day } & 0.312 & 0.27 & 4.11 & 36.13 & 2.85 & 0.223 & 1.137 & 0.649 & 0.61 \\
\hline \multicolumn{4}{|c|}{ SEm+ for SxD } & 0.273 & 0.24 & 3.60 & 31.6 & 2.496 & 0.195 & 0.995 & 0.567 & 0.538 \\
\hline \multicolumn{4}{|c|}{ F-test } & NS & NS & NS & & NS & & NS & $*$ & $*$ \\
\hline \multicolumn{4}{|c|}{ CD for $\mathrm{SxD}$} & - & - & - & 62.57 & - & 0.387 & - & 1.12 & 1.06 \\
\hline
\end{tabular}


Similarly, the seed colour changed from dark green to darkbrown at maturity (Table 2). The change in pod colour with the advancement of maturity was also reported by other researchers, also, in different tree species (Bonner, 1976; Ramakrishan et al., 1990; Bharathi et al., 1996; Rai et al., 1988 and Shah, 2005). Further, it has been observed that the pod dimensions i.e. length and width also increase with the advancement of colletion periods. With the advancement of maturity, the moisture content in pods also decreased. During the first collection in the month of February it was found maximum in all sites and thereafter it declined with the advancement of collection dates. At Agra site $\left(\mathrm{S}_{1}\right)$, the pod moisture content decreased from 52.9 to $40.6 \%$, whereas at Mathura $\left(S_{2}\right)$ and Hathrus sites $\left(\mathrm{S}_{3}\right)$ it decreased from 54.5 to $39.2 \%$ and $56.2 \%$ to $42.7 \%$, respectively (Table 1). The average pod moisture content at $\left(\mathrm{S}_{1}\right),\left(\mathrm{S}_{2}\right)$ and $\left(\mathrm{S}_{3}\right)$ was $54.2 \%, 56.1 \%$ and $57.7 \%$ at all three sites respectively (Table 1). Similarly, the seed moisture content also declined with the advancement of maturity.

Maximum seed moisture $\left(\mathrm{S}_{1} 53.1 \pm 0.39 \%, \mathrm{~S}_{2}\right.$ $53.1 \pm 0.32 \%$, and $\mathrm{S}_{3} 57.2 \pm 0.27 \%$ ) was observed in seeds collected during February month in all sites and the minimum $\left(\mathrm{S}_{1}\right.$ $42.2 \pm 0.47 \%, \quad S_{2} \quad 44.0 \pm 0.38 \%$, and $S_{3-}$ $45.9 \pm 0.38 \%)$ was in the seeds collected during $1^{\text {st }}$ week of June (Table 2). Loss of water during seed maturity is more inherent phase of seed development. The decline in seed moisture content during $\mathrm{pod} / \mathrm{seed}$ development is often attributed to the continued deposition of storage material in seeds (Ellis et al., 1987). Change in colour and decrease in moisture content were also identified as maturity indicators by many researchers (Grover et al., 1963; Carl and Snaw, 1971; Edward, 1980; Cram and Linquist, 1982; Welbaun and Bradford, 1988; Maideen et al., 1990; Singh, 1998 and Phyartyal et al., 2002). The mean pod/fruit length and width were recorded $20.26 \mathrm{~cm}$ and $1.85 \mathrm{~cm}\left(\mathrm{~S}_{1}\right), 20.18 \mathrm{~cm}$ and $2.0 \mathrm{~cm}\left(\mathrm{~S}_{2}\right)$ and $19.98 \mathrm{~cm}$ and $1.7 \mathrm{~cm}\left(\mathrm{~S}_{3}\right)$.

The data on seed germination (Table 2) revealed that the maximum germination $(58.7$ per cent at $S_{1}, 55.1$ per cent at $S_{2}$ and 54.2 per cent at $\mathrm{S}_{3}$ ) was recorded in the seeds collected during the $1^{\text {st }}$ week of June for all the sites. At this collection date, the pods and seeds were having brown and dark brown, respectively. At this stage the seed moisture content was $42.2 \% \mathrm{~S}_{1}, 44.0 \% \mathrm{~S}_{2}$ and $45.9 \% \mathrm{~S}_{3}$, the size of seeds was $46.6 \mathrm{~mm}^{2}\left(\mathrm{~S}_{1}\right), 44.0 \mathrm{~mm}^{2}\left(\mathrm{~S}_{2}\right)$ and $41.2 \pm 2.12 \mathrm{~mm}^{2}\left(\mathrm{~S}_{3}\right)$, seed weight/ 100 seeds was $152.7 \mathrm{~g}\left(\mathrm{~S}_{1}\right), 51.9 \mathrm{~g}\left(\mathrm{~S}_{2}\right), 50.9 \mathrm{~g}\left(\mathrm{~S}_{3}\right)$. Pandit et al., (2002) also reported the similar observation in Populus ciliata, the drop in moisture content of capsules from $80 \%$ to $60 \%$ during maturation coincided with the maximum germination in seeds.

From the present study, it may be concluded that colour as well as moisture content of pods and seeds are good indicators of maturity. First week of June is an appropriate period for pod collection for getting maximum germination in L. leucephalain semi-arid conditions of northern India.

\section{References}

Bharathi, A., UmaraniR., Karivaratharaju, T.V., Vanangamudi, K. and Manonmani, V. 1996. Effect of drup maturity on seed germination and seedling vigour in Neem. Journal of Tropical Forest Science. 9(2): 137-146.

Bonner, F.T. 1976 b. Maturation and collection of yellow poplar seeds in the mid South, Southern Forest Experiment Station. New Orleans, Lauisiana U.S.D.A. For. Serv. Res. Pap. 50-121, 8.

Carl, C.M. and Snow, Jr. A.G. 1971. Maturation of sugar maple seed. USDA Forest service, Research paper NE-217, Northestein Forest Experiment Station, Upper Darby, $\mathrm{Pa}$. 
Cram, W. H and Lindquist, C. H 1982. Germination of green ash (Fraxinus pennysylvanica) is related to seed moisture content at harvest. Forestscience, 28(4): 263-269.

Edwards, D.G.W. 1980. Maturity and quality of tree seeds of state of the art review. Seed Sci. and Technol., 8: 625-657.

Ellis, R.H. Hong, T.D. and Roberts, E.H. 1987. The development of desiccation tolerance and maximum seed quality during seed mathuration in six grain legumes. Ann. of Bot. 59: 23-29.

Grover, R. Martin, E.W. and Lindguist, C.H. 1963. Maturity and Storage of Siberian elm seed. For. Sci. 493-496.

I.S.T.A. 1981. Moisture content and equipment Wkg Group. In report of the Forest Tree Seed Committee. Seed Sci. and Technol. 20:29-32.

Kumar Naresh., Kumar Anil, Shukla, Ashok, Uthappa A. R., Prasad Rajendra and Ram Bahadur. 2018. Effect of arbuscular mycorrhizal inoculations on growth and seedling quality of four Leucaena species. Journal of Pharmacognosy and Phytochemistry. 7(4): 2089-2093

Lohani, D.N. 1979. Preliminary trial with high yielding varieties of Leucaena leucocephala; Indian Forester. 105(3): 199-200

Maideen, S.K. Selvaraj, J.A. and Vinayarai, R.S. 1990. Cone attributes as indices of seed maturity and effect of cone and seed grades on seed germination and vigour in casuarina sequisetifolia. J. R. \& G. Forst. Seed Sci. and Technol. 18: 483-489.

National Academy of Science. 1977. Leucaena: Promising forage and tree crop for tropics. National Academy of Science. Washington, D.C. 115.

Pandit, A., Pant, K. and Ram J. 2002. Effect of collection date on capsule moisture content and germination of Populus ciliata wallex. Rayole from Central Himalaya, New Forests. 23: 121-130.

Panse, V.G. and Sukhatame, P.V. 1961. Statistical method of Agriculture workers $2^{\text {nd }}$ Edn I.C.A.R. Book, New Delhi, India.

Parkash, Ram and Hocking Darake 1986. Some favourite trees for fuel and fodder, society for promotion of wasteland development, Sucheta Bhawan Annexe, 11-A Vishnu Digamber Marg, New Delhi,: 21-28.

Phartyal, S.S, Thapliyal, R.C; Nayal, J.S. and Joshi, G. 2000. Seed development in Himalyan elm (Ulmuswalli chiana) Seed science and Technology, 30(3): 575-584.

Rai. S.N., Nagaveni, H.C. and Ananthapadmanabha. H.S. 1988. Germination and Nursery technique of four species of ficus. Indian For. 114 (2): 6388.

Ramakrishnan, H.B., Jacqueline, A.S. and Vinaya Rai, R.S. 1990. Studies on ripeness index and presowing seed treatment in Ailanthus excels Roxb. Seed Sci. and technol. 18: 491-498.

Sajjan, A.S., Devaranavadi, S.B. and Honyal, S.C. 2000. Practical method of subabool (Leucaena lecocephala De. wit). Seed scarification. Advance in Foresry Research in India. 23: 160-164.

Shah. S., Tewari, B., Bisht. S. and Tewari. A. 2005 Seed maturation indicator in Pyracantha crenulata Roxb. in Kumaun Central Himalaya. New Forests, 32: 1-7.

Singh, O 1998. Seed maturity indices in Silver fir (Abies pindraw Spach). Indian Forester, 124(5): 243-246

Welbaun, G.E. and Bardford, K.J. 1988. Water relation of seed development and germination in Mushmelon (Cucumis melo L. I., Plant physiology, 86: 405-411.

\section{How to cite this article:}

Pankaj Lavania, Ashish Tiwari and Naresh Kumar. 2018. Studies on Maturity Indicators of Leucaena leucocephala in Northern India. Int.J.Curr.Microbiol.App.Sci. 7(12): 3636-3643. doi: https://doi.org/10.20546/ijcmas.2018.712.412 